\title{
Seizure Outcome in Adult Patients with Supratentorial Cavernomas
}

\author{
Rahsan Kemerdere ${ }^{1}$ Orkhan Alizada ${ }^{1} \quad$ Tugce Ayman ${ }^{1} \quad$ Oguz Baran ${ }^{2} \quad$ Seher Naz Yeni ${ }^{3}$ Taner Tanriverdi ${ }^{1}$ \\ ${ }^{1}$ Department of Neurosurgery, Medical Faculty, Istanbul \\ University-Cerrahpasa, Istanbul, Turkey \\ ${ }^{2}$ Department of Neurosurgery, Koç University Hospital, Istanbul, \\ Turkey \\ ${ }^{3}$ Department of Neurology, Medical Faculty, Istanbul \\ University-Cerrahpasa, Istanbul, Turkey \\ Address for correspondence Oguz Baran, MD, PhD, Department \\ of Neurosurgery, Koç University Hospital, Istanbul, Turkey \\ (e-mail: oguzbaran@gmail.com).
}

J Neurosci Rural Pract 2021;12:267-272.

\begin{abstract}
Keywords

- cavernoma

- cavernous angioma

- cavernous

hemangioma

- epilepsy

- seizure

Background Cavernomas are usually found in the supratentorial area, and epileptic seizures are one of the presenting symptoms.

Objective This study aims to provide the seizure outcome in adult patients who underwent surgical excision of single supratentorial cavernomas.

Materials and Methods A total of 23 patients with single supratentorial cavernomas were operated between May 2011 and January 2019. Pre- and postoperative seizure semiology, clinical, and radiological findings were collected from medical records. At the last follow-up, each patient was seen during regular visits and clinical variables were noted.

Results The mean age was $37.08 \pm 10.5$ years, and $11(57.8 \%)$ and $12(52.2 \%)$ were females and males, respectively. Headache $(43.5 \%)$ and seizure $(43.5 \%)$ were the most common presenting symptoms. Cavernomas were located on the right side in 13 and on the left side in 10 patients. The most common locations were the frontal (43.5\%) and temporal (43.5\%) lobes. The mean follow-up in this series was $41.4 \pm 30.8$ months. Our results showed that surgery was effective in seizure outcome, as almost $70 \%$ of patients who had seizure before surgery was seizure free after surgery, and the difference between those who had seizure pre- and postoperative periods was statistically significant $(p=0.0001)$.

Conclusion Surgery is safe and effective for supratentorial cavernomas. The excision of cavernoma together with the surrounding hemosiderin should be performed to obtain a satisfactory seizure outcome.
\end{abstract}

\section{Introduction}

Cavernomas, also known as cavernous hemangiomas or cavernous angiomas, account for approximately 10 to $15 \%$ of all vascular lesions of adult brain. ${ }^{1}$ Cavernomas are composed of enlarged, sinusoidal, and thin-walled blood vessels without intervening neural tissues, and they are angiographically occult lesions. The majority is found in the supratentorial area, and epileptic seizures are one of the presenting symptoms, manifesting at a rate of $70 \%{ }^{2}$ Diagnosis has become easier after the introduction of advanced magnetic resonance imaging (MRI) into the field. The importance of epileptic seizures in patients with supratentorial cavernomas is that almost $40 \%$ becomes medically refractory. ${ }^{3}$ Headaches and hemorrhage are also common, and the risk of hemorrhage is estimated at 3\% per person per year..$^{1,3,4}$ published online

November 12, 2020
DOI https://doi.org/

10.1055/s-0040-1721205 ISSN 0976-3147. (c) 2020. Association for Helping Neurosurgical Sick People.

This is an open access article published by Thieme under the terms of the Creative Commons Attribution-NonDerivative-NonCommercial-License, permitting copying and reproduction so long as the original work is given appropriate credit. Contents may not be used for commercial purposes, or adapted, remixed, transformed or built upon. (https://creativecommons.org/licenses/by-nc-nd/4.0/)

Thieme Medical and Scientific Publishers Pvt. Ltd., A-12, 2nd Floor, Sector 2, Noida-201301 UP, India 
Several studies documented the clinical and radiological features of cavernomas, but there is still a controversy whether they should be treated conservatively with antiepileptic drugs (AED) with respect to seizures or whether resective surgery is mandatory. ${ }^{1,3,4}$ The available data in the current literature show that there is no doubt that surgery should be performed on symptomatic cavernomas to prevent future bleeding and refractory seizure. ${ }^{5-9}$ Many previous studies reported relatively good surgical and seizure outcomes and emphasized the importance of early surgery. ${ }^{5-9}$ Another controversy that is still ongoing is the extent of excision. As far as we know, there are three main types of surgical excisions for cavernomas: excision of the lesion only (excision of cavernomas without the surrounding hemosiderin), lesionectomy (excision of cavernomas together with the surrounding hemosiderin), and extended lesionectomy (excision of 1 or $2 \mathrm{~cm}$ beyond the cavernomas and the surrounding hemosiderin). Comparative studies have demonstrated that lesionectomy is superior to the excision of lesions only in patients with seizure, and lesionectomy is sufficient to obtain a favorable seizure outcome. ${ }^{5.8}$ Extended lesionectomy has been suggested to patients who show medically intractable epilepsy, and whose preoperative diagnostic work-up such as electroencephalography (EEG) has proved that the epileptic zone is more extensive than the cavernoma and the surrounding hemosiderin., ${ }^{5,7}$ The most challenging part of surgery is encountered when a cavernoma is located in or close to the eloquent area. In these situations, awake craniotomy with cortical electric stimulation seems to be rational.

This study, which includes a retrospective cohort of adult patients who underwent surgery on supratentorial cavernomas, aims to share our experience in surgical (and seizure) outcome with the readers. All surgeries were performed by the same surgeon with the same surgical technique (lesionectomy: excision of the cavernoma together with the surrounding hemosiderin rim).

\section{Materials and Methods}

\section{Patients}

This retrospective study was approved by the local ethics committee and does not include any data disclosing the patients' identity. Between May 2011 and January 2019, a total of 31 patients underwent cavernoma surgery. Six patients who were lost to follow-up, one patient with cerebellar cavernoma, and one with multiple supratentorial cavernomas were excluded from the data. Ultimately, 23 patients with single supratentorial cavernomas were included and analyzed in this study.

\section{Methods}

Data included pre- and postoperative variables: age at surgery, sex, presenting symptom, presence of seizure, type and frequency of seizure, findings from neurologic examinations, MRI features (site, side, and presence of hemosiderin rim), number of AED used, findings from EEG if obtained, and surgical complications if present. Seizure frequency was divided into two groups those who had only one seizure and those who experienced more than one seizure until surgery. All patients had pre- and postoperative head MRI with all sequences assessed by our experienced neuroradiologists. Seizure outcome was divided into two groups those who had seizure and those who were completely seizure free since surgery. Follow-up data were obtained during patients' regular visits or by phone calls. All patients provided early (within 72 hours of surgery) MRI after surgery and at the 6-month follow-up. Lesionectomy (excision of cavernoma together with the surrounding hemosiderin rim) was our main surgical strategy. If the cavernoma was located in a safe zone ( $\boldsymbol{- \text { Fig. }} \mathbf{1}$ ), the surgery would be straightforward: lesionectomy without hesitation. However, in the case of the cavernoma being close to or located in an eloquent area (-Fig. 2), awake craniotomy was assisted by cortical electric stimulation, navigation would be performed, and the hemosiderin rim would be removed as much as possible. In the
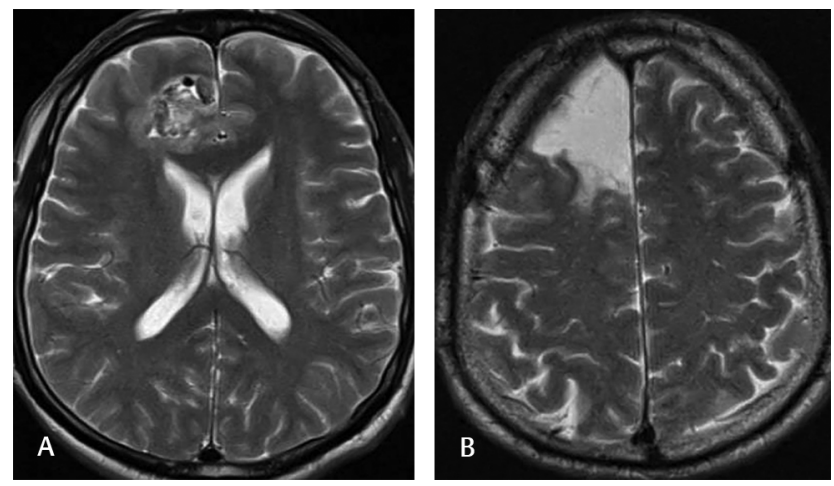

Fig. 1 This 55-year-old male patient was admitted to our clinic with a complaint of multiple generalized tonic-clonic seizures. Neurological examination was normal, and the patient was not using any AED. Scalp EEG showed seizure activity from the right frontal lobe. The preoperative $\mathrm{T}_{2}$-weighted MRI (A) demonstrated a mulberry-shaped cavernoma surrounded by a hemosiderin rim. The patient underwent lesionectomy, and the postoperative $\mathrm{T}_{2}$-weighted MRI (B) showed no lesion. The patient had only one seizure since surgery (at 1-year follow-up) and was on AED. AED, antiepileptic drug; EEG, electroencephalography.
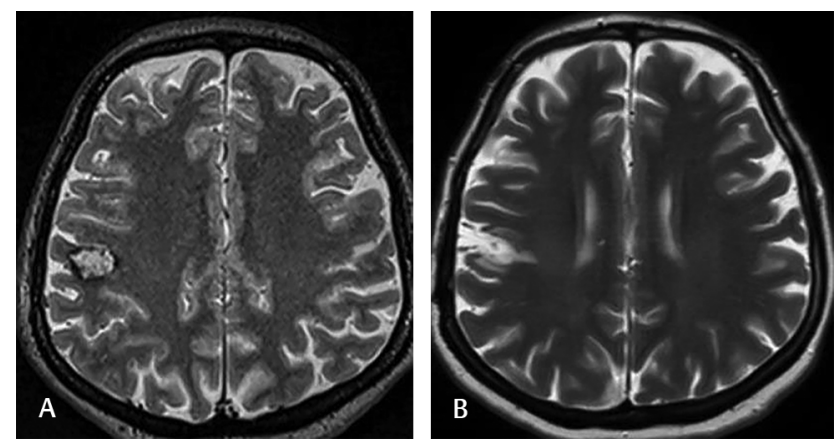

Fig. 2 This 40-year-old female complained of numbness on the left side of her face. Preoperative $T_{2}$-weighted MRI (A) showed cavernomas with a surrounding hemosiderin rim close to the right motor strip. The patient underwent awake craniotomy with cortical electric stimulation and navigation. Postoperative $\mathrm{T}_{2}$-weighted MRI (B) at the 2-year follow-up demonstrated no lesion. The patient was doing well without any medication. MRI, magnetic resonance imaging. 

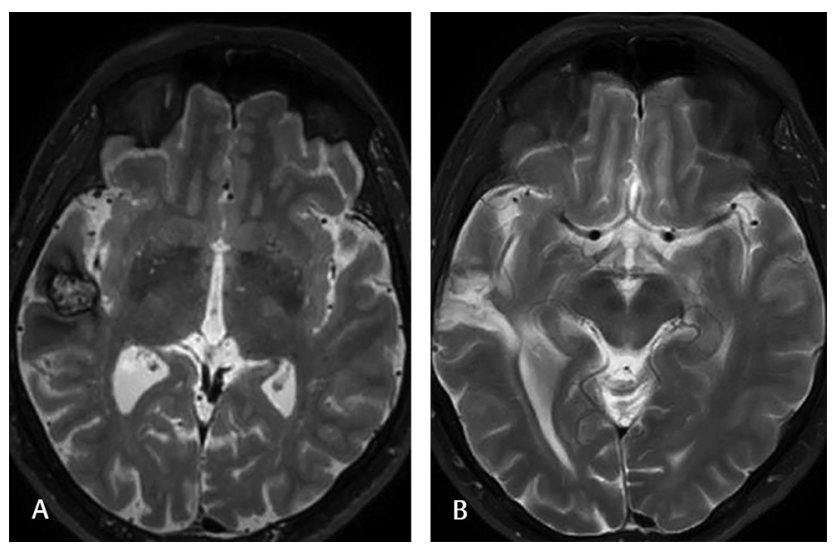

Fig. 3 This 25-year-old male experienced several seizures before surgery. Scalp EEG showed seizure activity from the right temporal lobe. Head $\mathrm{T}_{2}$-weighted MRI (A) demonstrated a cavernoma with a hemosiderin rim on the right temporal cortex. The patient underwent lesionectomy. The postoperative $\mathrm{T}_{2}$-weighted head MRI (B) at the 6-month follow-up showed no lesion. The patient experienced only one seizure and was still on AED. AED, antiepileptic drug; EEG, electroencephalography.
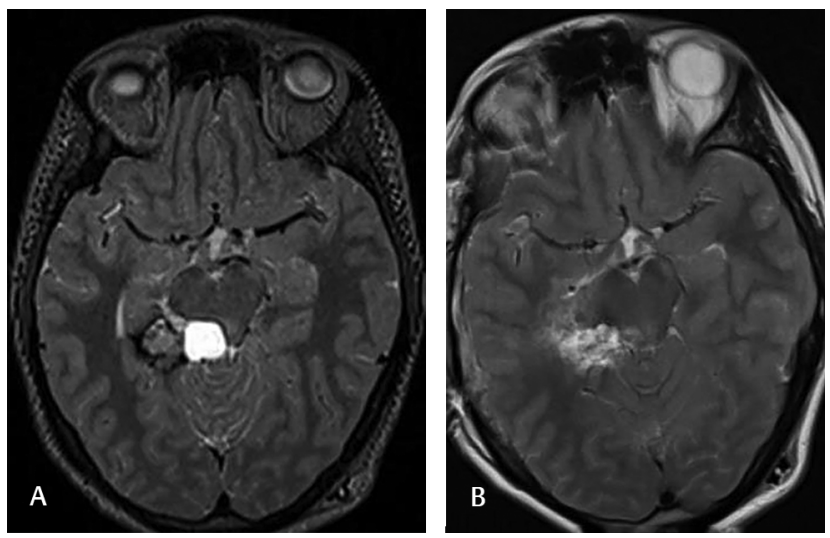

Fig. 4 This 26-year-old female complained of headaches, and the preoperative $\mathrm{T}_{2}$-weighted MRI (A) showed a cavernoma located on the right posterior hippocampal-parahippocampal complex. The patient underwent lesionectomy. The postoperative $\mathrm{T}_{2}$-weighted MRI (B) at the 6-month follow-up showed no lesion. The patient was doing well without any neurological sequela.

temporal area, we divided the location into two zones: temporal cortical (lateral to the collateral sulcus; - Fig. 3) and temporal mesial (medial to the collateral sulcus; - Fig. 4). We did not perform the whole mesial temporal resection in the case of medially located small- or medium-sized cavernomas. In the case of a cavernoma located in the temporal neocortex, we did not perform an extended resection, and lesionectomy was again performed. Data from all EEGs were hoped to be consistent with the topographic location of the cavernoma in patients with seizure.

\section{Statistical Analysis}

We used a commercially available statistical software package (SPSS version 22.0; IBM Corporation, Inc., Chicago, Illinois, United States) for all statistical analyses. The mean \pm standard deviations were calculated for each parameter.
Chi-square tests were used for appropriate comparisons. Differences were considered statistically significant if the probability value was less than 0.05 .

\section{Results}

- Table 1 summarizes some clinical and radiological features of the 23 patients. Among the 23 patients, the mean age was $37.08 \pm 10.5$ years (range: $20-55$ years), and 11 (57.8\%) and $12(52.2 \%)$ were female and male, respectively. No significant difference was detected in terms of sex $(p=08)$. Headache ( $n=10 ; 43.5 \%)$ and seizure $(n=10 ; 43.5 \%)$ were the most common presenting symptoms. Among the 10 patients with preoperative seizures, generalized tonic-clonic and complex partial seizures were observed in five (50\%) and five (50\%) patients, respectively. Five (50\%) patients had only one seizure, and the remaining five (50\%) experienced more than one seizure until surgery. Scalp EEG was performed on only five patients, and it was expected to reveal a unifocal finding with a topographic relation to the cavernoma. Interestingly, only $4(40 \%)$ of the 10 patients with preoperative seizures were on AED when the patients were seen by our team, and all were on monotherapy. Abnormal neurological examinations were found in only two patients (8.7\%): one had drowsiness and the other showed motor weakness in both lower extremities.

All patients had MRI with 1.5 or 3T with all sequences. Aside from head MRI, our center also routinely performs digital subtraction angiography to determine the presence or absence of any venous anomaly that may be associated with cavernomas. The head MRI showed that the cavernomas were located on the right side in 13 (56.5\%) and on the left side in 10 (43.5\%) patients, and that no significant difference was found in terms of location side $(p=0.53)$. The most common locations were the frontal $(n=10 ; 43.5 \%)$ and temporal ( $n=10 ; 43.5 \%$ ) lobes, followed by the occipital $(n=2 ; 8.7 \%)$ and parietal $(n=1 ; 4.3 \%)$ lobes. All cavernomas were single and had a hemosiderin rim surrounding them. Among the five temporal cavernomas, two were located medial to the collateral sulcus: one in the uncus and the other in the posterior hippocampal-parahippocampal complex. In five patients $(21.7 \%)$, the cavernoma was located deeply in the white matter or in deep structures. Two were mesial to the collateral sulcus in the temporal lobe as mentioned above. In the remaining three, one was in the cingulate gyrus, one was in the white matter close to the basal ganglia, and one was in the occipital lobe white matter. All patients had early MRI within 72 hours of surgery, and lesionectomy was shown to be accomplished in all MRI findings.

As the lesionectomy, which is the removal of the cavernoma together with the surrounding hemosiderin rim, we performed in this study has been described in the literature, there is no need to explain it in detail here. We simply emphasize that we did not hesitate to perform lesionectomy in all cavernomas, even those close to the eloquent area. In this series, only three cavernomas located close to the motor strip (two patients) and basal ganglia (one patient) required awake craniotomy with cortical electric stimulation. 
Table 1 Summary of clinical and radiological characteristics of the patients

\begin{tabular}{|c|c|c|c|c|c|c|c|c|}
\hline No & Age & Sex & Symptom & Finding & Side & Site & Complication & ${ }^{\mathrm{a}} \mathrm{F} / \mathrm{U}$ \\
\hline 1 & 20 & $\mathrm{~F}$ & Cog. Dec. & None & Left & Frontal & None & 101 \\
\hline 2 & 30 & $\mathrm{~F}$ & Headache & None & Left & Temporal & None & 84 \\
\hline 3 & 38 & $\mathrm{M}$ & Headache & None & Right & Temporal & None & 88 \\
\hline 4 & 24 & M & Headache & None & Left & Frontal & None & 82 \\
\hline 5 & 38 & $M$ & Seizure & None & Right & Frontal & None & 82 \\
\hline 6 & 49 & $\mathrm{~F}$ & Headache & None & Left & Temporal & None & 80 \\
\hline 7 & 51 & $\mathrm{~F}$ & Seizure & None & Left & Frontal & None & 76 \\
\hline 8 & 35 & $\mathrm{~F}$ & Headache & Drowsy & Right & Frontal & None & 50 \\
\hline 9 & 55 & $\mathrm{M}$ & Seizure & None & Right & Frontal & $\mathrm{EDH}$ & 26 \\
\hline 10 & 40 & $\mathrm{~F}$ & bNumbness & None & Right & Frontal & None & 24 \\
\hline 11 & 46 & $M$ & Headache & None & Right & Parietal & None & 23 \\
\hline 12 & 26 & $\mathrm{~F}$ & Headache & 'Weakness & Right & Temporal & None & 20 \\
\hline 13 & 21 & $\mathrm{~F}$ & Seizure & None & Left & Temporal & None & 21 \\
\hline 14 & 49 & $\mathrm{~F}$ & Seizure & None & Right & Temporal & None & 19 \\
\hline 15 & 36 & M & Headache & None & Left & Occipital & CSF fistula & 12 \\
\hline 16 & 34 & $\mathrm{M}$ & Headache & None & Right & Temporal & None & 12 \\
\hline 17 & 54 & $\mathrm{M}$ & Seizure & None & Right & Temporal & None & 10 \\
\hline 18 & 32 & $\mathrm{~F}$ & 'Weakness & None & Left & Frontal & None & 10 \\
\hline 19 & 36 & $\mathrm{~F}$ & Seizure & None & Left & Frontal & None & 36 \\
\hline 20 & 25 & M & Headache & None & Right & Occipital & None & 23 \\
\hline 21 & 43 & $\mathrm{M}$ & Seizure & None & Left & Temporal & None & 36 \\
\hline 22 & 44 & $\mathrm{M}$ & Seizure & None & Right & Frontal & None & 23 \\
\hline 23 & 27 & M & Seizure & None & Right & Temporal & None & 12 \\
\hline
\end{tabular}

Abbreviations: CSF, cerebrospinal fluid; Cog. Dec., cognitive decline; EDH, epidural hematoma; F, female; M, male.

aF/U: follow-up (months).

bNumbness: feeling of numbness on the left hand.

'Weakness: feeling of weakness on both lower extremities.

'Weakness: motor weakness on both lower extremities (4/5).

After surgery, early neurological examinations revealed that only one patient had left hemiparesis after temporal cavernoma surgery and it was transient. During the long-term follow-up, none of the patients had neurological deficits. Surgery-related complications were observed in two patients (8.7\%). One patient had an epidural hematoma following right frontal surgery, and no surgery was required for this complication. The second patient had cerebrospinal fluid fistula, which required surgery, and thus, dural repair was performed. The postoperative course was uneventful, and the patient was discharged from the hospital without further clinical problems.

The mean follow-up in this series was $41.4 \pm 30.8$ months (range: 10-101 months). During the last follow-up or last visit, all patients were neurologically intact. Among the 10 patients with preoperative seizures, only three patients (30\%) had seizures after surgery. Two patients and one patient were operated on the right temporal and left frontal cavernomas, respectively. One had seizure three times since surgery (20-month follow-up), and the other two only had one seizure (one in 3 years and one in the 10-month follow-up). We decided to continue the AED in these three patients, who were closely followed-up by our team to stop the AED in the future. Our results showed that surgery was very effective in seizure outcome, almost $70 \%$ of patients who had seizure before surgery was seizure free after surgery, and the difference between those who had seizure pre- and postoperative periods was statistically significant $(p=00001)$. At the last follow-up, several patients informed us that they sometimes had headaches, which were not severe and did not decrease their quality of life.

\section{Discussion}

Common symptoms and/or findings in patients with supratentorial cavernomas include headaches, microhemorrhages, focal neurologic deficits, and epileptic seizures. The current literature ${ }^{5,7,9,10}$ and our own findings show that epileptic seizures are the most common presenting symptoms. Therefore, it can be accepted now that supratentorial cavernomas with epileptic seizures have become the focus of interest of epileptologists and epilepsy surgeons. Seizures secondary to supratentorial cavernomas should be evaluated in detail in epilepsy patients before surgery, as defining the 
epileptic zone is of utmost importance given that in almost $6 \%$ of patients with supratentorial cavernomas, the focus of seizure is not related to the cavernoma itself. ${ }^{11}$ The continuation of seizure after the excision of cavernoma may be detrimental to patients and decrease their quality of life. This important issue explains why studies in the current literature have mainly focused on seizure in supratentorial cavernomas.

The exact pathophysiology behind seizures in supratentorial cavernomas has been considered complex and not fully understood. However, an increasing number of studies have indicated that microhemorrhages caused by cavernoma may be responsible for seizures. Microhemorrhages lead to the accumulation of hemosiderin, a degradation product of ferritin, which is the focus of epilepsy., ${ }^{5,79}$ The deposition of hemosiderin and reactive gliosis around a cavernoma may influence the microenvironment where a newly formed synapsis and an increased number of excitatory amino acids may contribute to the development of epilepsy. ${ }^{12}$ There is a common notion that cavernoma itself has no intrinsic epileptogenic activity, as there is no neuronal tissues within the cavernoma. Furthermore, it has been suggested that a mass effect caused by cavernoma may not explain refractory epilepsy because other large lesions, such as malignant gliomas, are less commonly associated with medically refractory epilepsy. ${ }^{13}$ Under this premise, it is obvious that the excision of lesions without the surrounding hemosiderin seems insufficient for favorable seizure outcome, and lesionectomy together with the surrounding hemosiderin is probably necessary.

On the basis of the present results, we recommend that hemosiderin should be removed with the cavernoma, and our results on seizure outcome are consistent with the current literature ${ }^{5-7,9,10}$ that a long-term seizure-free rate was found to be $70 \%$ in our patients. Only three patients had seizures during the follow-up period, and their seizure frequency significantly decreased. Furthermore, we agree with some studies that hemosiderin rim can be safely removed in cavernomas close to the eloquent area with the assistance of navigation and cortical electric stimulation, given that functional MRI and intraoperative mapping studies have shown that the surrounding hemosiderin is not embedded in the functional cortex..$^{14}$ In this study, we did not have a chance to evaluate the prognostic factors for seizure outcome mainly because of the low number of patients. However, we can speculate that the location of the lesion did not influence seizure control.

There is still a debate on what should be the limit of excision in patients with seizure secondary to supratentorial cavernoma. Some authors supported extended lesionectomy including the epileptogenic cortex, ${ }^{7,14}$ but others reported that lesionectomy including the cavernoma and the surrounding hemosiderin is enough to obtain a satisfactory seizure outcome..$^{5,6,9,15}$ The results of a recent meta-analysis ${ }^{16}$ showed no significant difference in seizure outcome between extended lesionectomy and lesionectomy, and that the resectioning of the cavernoma and the surrounding hemosiderin is sufficient for patients with a cavernoma with epilepsy. We agree with the majority of studies that lesionectomy is sufficient for a cavernoma with seizure, and extended lesionectomy may lead to a severe neurologic deficit in a cavernoma close to an eloquent area. Our main surgical strategy in supratentorial cavernoma in any location, including deep-seated or white matter-seated, is lesionectomy (excision of the cavernoma with the surrounding hemosiderin), which is safe and effective in terms of seizure outcome and other clinical outcome such as headaches. Another issue we faced during surgery was patients with temporal lobe cavernomas. Previous studies supported the view of extended resectioning, such as the resectioning of the temporal cortex in case of a cavernoma located lateral to the collateral sulcus or the resectioning of the mesial temporal lobe or temporal lobectomy in case of a cavernoma located medial to the collateral sulcus. ${ }^{17,18} \mathrm{We}$ did not perform extended temporal resections in temporal lobe cavernomas in the present series. We think that the two patients with temporal cavernomas who had seizures during the long-term follow-up period deserve to be mentioned here. Both patients had a cavernoma located in the temporal cortex. They suffered only one seizure after surgery, and at the last follow-up, they were seizure free. Seven patients (70\%) of the 10 with a temporal cavernoma were completely seizure free since surgery. Thus, unlike in some previous studies, our data did not support the view that extended resectioning of temporal lobe cavernomas is more effective.

Another important point that we want to emphasize is that only four patients among the 10 who had seizures before surgery were on AED when they were seen by our team. We strongly encourage the referred physicians to start AED in patients with a cavernoma with seizure. Our observations indicated that many patients would be seeing several physicians, and the patients were informed that a cavernoma was not a tumor, surgery was not mandatory even with seizure, and AED would not be prescribed. This information could have caused patients to delay admission to advanced surgery clinics, and thus, they continued to have seizures. We emphasize that repetitive seizures may become refractory with time, and that an effective treatment, such as surgery, should be performed as soon as possible to prevent further hemorrhage and refractory seizures.

\section{Study Limitations}

The retrospective design, limited number of patients, and relatively short follow-up are the main limitations of the study. Several questions related to surgical techniques can only be answered by performing a prospective study design with a larger patient cohort in the future.

\section{Conclusion}

Surgery is safe and effective in supratentorial cavernomas. The excision of a cavernoma together with the surrounding hemosiderin should be performed to obtain a satisfactory seizure outcome. Deep-seated cavernomas or cavernomas close to an eloquent area can be safely removed by conducting awake craniotomy with the assistance of navigation and cortical electric stimulation. 


\section{Funding}

None.

\section{Conflict of Interest}

None declared.

\section{References}

1 Batra S, Lin D, Recinos PF, Zhang J, Rigamonti D. Cavernous malformations: natural history, diagnosis and treatment. Nat Rev Neurol 2009;5(12):659-670

2 Kraemer DL, Awad IA. Vascular malformations and epilepsy: clinical considerations and basic mechanisms. Epilepsia 1994;35(Suppl 6) :S30-S43

3 Kondziolka D, Lunsford LD, Kestle JR. The natural history of cerebral cavernous malformations. J Neurosurg 1995;83(5): 820-824

4 Del Curling O Jr, Kelly DL Jr, Elster AD, Craven TE. An analysis of the natural history of cavernous angiomas. J Neurosurg 1991; 75(5):702-708

5 Baumann CR, Acciarri N, Bertalanffy H, et al. Seizure outcome after resection of supratentorial cavernous malformations: a study of 168 patients. Epilepsia 2007;48(3):559-563

6 Daglioglu E, Ergungor F, Polat E, Nacar O. Microsurgical resection of supratentorial cerebral cavernomas. Turk Neurosurg 2010; 20(3):348-352

7 von der Brelie C, Malter MP, Niehusmann P, Elger CE, von Lehe M, Schramm J. Surgical management and long-term seizure outcome after epilepsy surgery for different types of epilepsy associated with cerebral cavernous malformations. Epilepsia 2013;54(9):1699-1706

8 Wang X, Tao Z, You C, Li Q, Liu Y. Extended resection of hemosiderin fringe is better for seizure outcome: a study in patients with cavernous malformation associated with refractory epilepsy. Neurol India 2013;61(3):288-293
9 Yeon JY, Kim JS, Choi SJ, Seo DW, Hong SB, Hong SC. Supratentorial cavernous angiomas presenting with seizures: surgical outcomes in 60 consecutive patients. Seizure 2009; 18(1):14-20

10 Khallaf M, Abdelrahman M. Supratentorial cavernoma and epilepsy: experience with 23 cases and literature review. Surg Neurol Int 2019;10:117

11 Requena I, Arias M, López-Ibor L, et al. Cavernomas of the central nervous system: clinical and neuroimaging manifestations in 47 patients. J Neurol Neurosurg Psychiatry 1991; 54(7):590-594

12 Seifert G, Schilling K, Steinhäuser C. Astrocyte dysfunction in neurological disorders: a molecular perspective. Nat Rev Neurosci 2006;7(3):194-206

13 Schuele SU, Lüders HO. Intractable epilepsy: management and therapeutic alternatives. Lancet Neurol 2008;7(6):514-524

14 He K, Jiang S, Song J, Wu Z, Chen L, Mao Y. Long-term outcomes of surgical treatment in 181 patients with supratentorial cerebral cavernous malformation-associated epilepsy. World Neurosurg 2017; 108:869-875

15 Jin Y, Zhao C, Zhang S, Zhang X, Qiu Y, Jiang J. Seizure outcome after surgical resection of supratentorial cavernous malformations plus hemosiderin rim in patients with short duration of epilepsy. Clin Neurol Neurosurg 2014;119:59-63

16 Shang-Guan HC, Wu ZY, Yao PS, Chen GR, Zheng SF, Kang DZ. Is extended lesionectomy needed for patients with cerebral cavernous malformations presenting with epilepsy? A meta-analysis. World Neurosurg 2018;120:e984-e990

17 Englot DJ, Han SJ, Lawton MT, Chang EF. Predictors of seizure freedom in the surgical treatment of supratentorial cavernous malformations. J Neurosurg 2011;115(6):1169-1174

18 Kim W, Stramotas S, Choy W, Dye J, Nagasawa D, Yang I. Prognostic factors for post-operative seizure outcomes after cavernous malformation treatment.J Clin Neurosci 2011;18(7): 877-880 